\title{
Recent Advances in Finishing and Polishing for Restorative Materials - A Review
}

\author{
Shweta Gupta ${ }^{1}$, Parinitha Mysore Shankar², Sowmya Halassabalu Kalgeria ${ }^{3}$, Ashwini Tumkur Shivakumar ${ }^{4}$ \\ 1,2, 3, 4 Department of Conservative Dentistry and Endodontics, \\ JSS College of Dental Sciences and Hospital, Mysuru, India.
}

\section{ABSTRACT}

The important key to dentistry is to render best dental care to the patients. Science is undergoing great revolutions that are leading us to a new era of dentistry. The development and implementation of any new system relies on comprehensive understanding of the existing systems. A well contoured, finished, and polished restoration results in welfare regarding dental concern in terms of oral health, oral function and aesthetics. These procedures call for an incremental stepwise approach establishing finest scratches to the substrate surface in order to systematically eliminate the deeper or profound scratches from previous grinding.

Fortunately, dentistry has been the focal point of a great covenant research in recent years with the desire of ameliorating restoration performances. In this article we put forward the basic foundation regarding the finishing polishing technique, the ideology behind it, objectives and why it is important. The article discusses evidence based information to enlighten the paramount significance of this step in enhancing the properties of a restoration.

Here, in this article we majorly review the recent aids or inventions made in the field of finishing and polishing materials with currently published reference articles. The knowledge and impact of new technologies is higher than we expected. It helps us in improvising our methodologies with enlightening places of correlation with research. It helps us improve our work and deliver high quality treatment with less time and more patient satisfaction. The overall point of this article is to give the readers with an improved mindfulness and more extensive information on the standards and apparatuses accessible to create ideal dental restorations.

\section{KEY WORDS}

Abrasives, Dental Restorations, Finishing, Polishing, Recent Advances
Corresponding Author: Dr. Shweta Gupta, Department of Conservative Dentistry and Endodontics, Room No.7, First Floor, JSSDCH, SS Nagar, Bannimantap, Mysuru, Karnataka - 570015, India. E-mail: gupta.shweta0226@gmail.com

DOI: $10.14260 /$ jemds/2021/578

How to Cite This Article:

Gupta S, Shankar PM, Kalgeri SH, et al. Recent advances in finishing and polishing for restorative materials - a review. J Evolution Med Dent Sci 2021;10(33):28412846, DOI: $10.14260 /$ jemds/2021/578

Submission 31-01-2021,

Peer Review 14-06-2021,

Acceptance 21-06-2021,

Published 16-08-2021.

Copyright (C) 2021 Shweta Gupta et al. This is an open access article distributed under Creative Commons Attribution License [Attribution 4.0 International (CC BY 4.0)] 


\section{BACKGROUND}

Finishing and polishing of dental restorations are principal ingredients of clinical procedures that supplement both aesthetics and durability. ${ }^{1}$ Remnant surface irregularity corresponding to improper finishing and polishing can result in accumulation of plaque, irritation of gingival epithelium, and increased surface staining producing poor or suboptimal aesthetics. ${ }^{2}$ Finishing and polishing are procedures that are both based on the principle of wear activity, but they differ in their end purpose and degree. ${ }^{3}$ These procedures call for an incremental stepwise approach establishing finest scratches to the substrate surface in order to systematically eliminate the deeper or profound scratches from previous grinding.

The primary objective of a dental specialist is to reinstate the individual tooth close to the form and functions of that of an original tooth structure alongside imparting gratifying aesthetics and maintaining periodontal tissue condition in great regard. It has been stated and reported in literature that rough or uneven surfaces act as active substrates for microbial flora to initiate and flourish. Furthermore, the light reflected by these surfaces may not be even and uniform. ${ }^{2,4}$ Hence a restored tooth structure should be smooth evenly and reflect light uniformly which is obtained through finishing and polishing. Finishing is defined as the transformation of an object from a rough to a refined form. The technique includes expulsion of surface irregularities, shaping and constructing a restoration according to the fundamentals of occlusion. Polishing is defined as the production of a shiny mirror like surface, which reflects light similar to enamel and reduction in roughness and scratches typically created by finishing instruments. 5,6

Many steps go into the success and longevity of a restoration out of which finishing and polishing are considered critical steps to decrease plaque accumulation and prevent gingival irritation, secondary caries and discoloration of restorative materials. ${ }^{7-11}$ These systems bring about reduction in wear and add to the marginal and periodontal integrity thus enhancing the clinical life span of dental restorations. ${ }^{12-14}$ Several techniques have been standardized and utilized in order to measure a surface for its roughness. Profilometer, an optical microscope or a Scanning Electron Microscope that can be considered as one of the few standard instruments used to assess the quality of the surface finish and lustre by the estimation of surface roughness. ${ }^{15}$ The mechanism behind finishing and polishing is to utilize a sequence of coarse articles or abrasives to remove material at micro level from a comparatively softer body to an extent that the produced surface scratches by each abrasive are successively eliminated with ones of smaller dimension.

\section{Importance of Finishing and Polishing}

- It guarantees the oral well-being and longer life span of restorations. A shiny glassy well-polished surface diminishes the probability of adherence, implying that plaque is more averse to aggregate on a cleaned surface. This promotes better, longer enduring restorations. ${ }^{16,17}$

- A well smoothened and polished tooth is more organically viable with the gingival tissue, so the health and soundness of the surrounding gingival tissue is maintained and preserved. Such teeth require less maintenance and are simpler to keep up in a clean hygienic state with day-to-day oral hygiene practices.

- Proper contouring, finishing, and polishing will strengthen the marginal integrity of the restoration especially the ones that are under tension. Interproximally the potential for plaque retention is reported to be the highest and polishing these areas will significantly reduce the likelihood of secondary caries and diseases related to the periodontium.

- An exceptionally polished tooth surface results in more natural enamel - like lustre creating aesthetic smiles. This is due to the increase in the reflective and refractive index properties of a restoration. So from an aesthetic point of view, a restoration simply cannot be left unpolished.

- Tarnish and corrosion, an activity seen with certain dental materials can be significantly reduced or even prevented if the entire restoration is highly polished.

- $\quad$ Rough materials tend to act as stress concentrating areas that can result in loss of stable and functional contacts within the occlusion. If properly followed, finishing and polishing greatly enhances the longevity, durability, and long-term wear resistance of the restoration.

- As aesthetics has become the prime concern these days, finishing and polishing enhances patient comfort and satisfaction, and patients greatly appreciate the natural aesthetical compatibility and health benefits acknowledged from an appropriately polished restoration. 18

\section{BASIC PRINCIPLES OF FINISHING AND POLISHING}

The principles involved in mechanical finishing and polishing using abrasive particles are based on the concept of tribology which is the discipline associated with material science, physics, chemistry, and surface - contact engineering. This concept is a combination of experimental parameters (e.g., applied load, velocity, and duration of motion) and the system structure (e.g., the two - body contact, the interfacial media, and the surrounding media). ${ }^{12}$ Finishing and polishing tools, mediums, and methods are intended to execute a specific, intentional, and guided wear of the restorative material surfaces..$^{13}$ Wear is defined as a cumulative surface damage in which the material is removed from a body as small debris particles, mainly by mechanical processes. The wear mechanism is the transfer of energy with removal or displacement of material. ${ }^{14}$ The four wear mechanisms are adhesion, abrasion, surface fatigue, and tribochemical reactions. ${ }^{12}$ The ultimate goal is to achieve the desired anatomical features, a balanced occlusion, and the decrease in roughness, gouges and scratches that were formed during the preparation phase.

\section{Objectives}

Finished and polished restorations result in four advantages of dental / oral care: healthy gums / gingiva, better efficiency in chewing, patient comfort, and improved aesthetics. The 
objectives of finishing and polishing of any restoration is to obtain a satisfactory fit and continuity in margins, optimal contour and contacts and a scratch or irregularity free surface to make it biologically acceptable. These objectives together establish the framework of an ideal oral well-being and aesthetics. ${ }^{19,20,21}$

\section{Oral Health}

A skilfully defined and burnished restoration will have a decreased surface area and reduced roughness of the restoration surface which promotes the oral health by resisting the accumulation of food debris and pathologic bacteria. Smoother surfaces are easier to maintain in a hygienic state when preventive oral care is practiced. With some restorations, tarnish and corrosion activity can be significantly reduced if the entire restoration is highly polished, which is very important for the biocompatibility property of a material.

\section{Oral Function}

It is amplified with a well burnished restoration because food glides more freely over well contoured occlusal and embrasure surfaces during mastication. Smooth restoration contacts minimize wear rates on opposing and adjacent teeth, which is particularly true for restorative materials such as ceramics that contain phases that are harder than enamel and dentin. Friction is defined as the resistance to motion of one body over another and a restraining force is produced to resist this motion. This phenomenon can be reduced if the surfaces are glossy or smooth and found in a single plane. Reduced friction results in reduced wear which ultimately increases the life span of the existing restoration. Irregularities or roughness on material surfaces attract high-contact stresses and hence development of these stresses results in the loss of functional and stabilizing contacts between teeth.

\section{Aesthetics}

With the increase in aesthetic demands the clinician is required to emphasize and prepare highly visible surfaces of restorations differently as compared to those that have least accessibility. When a solid is exposed to white light, a minor proportion of this light is reflected directly from the surface and hence remains white. This in turn gets mixed with the existing light and causes a dilution of the colour. An application of this phenomenon is seen in the case of an extremely rough surface which appears to be lighter in contrast to a smoother surface of the same material. This drawback is noticed with unpolished or sapped glass ionomer cement (GIC) and resin composite restorations.

Opacity, translucency, and transparency are three characteristic features of a dental material that are affected by the surface quality of the material. Opacity is a property of materials that obstructs the light from passing. An opaque surface absorbs some of the light and reflects the remainder. An example of this is porcelain, which is opaque and hence reflects yellow light resulting in yellowish appearance. Translucency is another property that also obstructs light transmission but causes dispersion of the light, because of which items can't be seen through the material. Examples of translucent materials used in dentistry are ceramics, resins, composites etc. Transparent materials allow complete light transmission with little distortion and objects appear clearly when seen through these materials.

\section{RECENT ADVANCES IN FINISHING AND POLISHING}

Evolving methods and technologies offer clinicians new tools to meet patient demand and satisfaction for quality delivery. While most recent products in the dental finishing and polishing arena are added upon contemporary products, welcoming new ideas, blueprints, or materials ultimately improve and broaden the access to dental care and periodically raises greater interest. This review article focuses on representing the recent concepts of finishing and polishing currently in research or those that require further evaluation and needs more focus and limelight in the current practice.

\section{Air-Particle Abrasion Technology}

As an alternative to the use of rotary instrument, air-particle abrasive systems are minimal invasive technologies that can deliver a definitely controlled high - pressure fine stream of 25 $\mu \mathrm{m}$ to $30 \mu \mathrm{m}$ aluminium oxide $\left(\mathrm{Al}_{2} \mathrm{O}_{3}\right)$ or silica particles to remove decayed or stained enamel, dentin, and restorative materials. Because air particle abrasion generates minimal heat and vibration, there is relatively little potential for tooth chipping or microfracturing. ${ }^{22}$ These systems have been used for the following applications -

- tooth preparation for receiving any restoration,

- removal of defective restorations such as resin-based composite,

- access cavity through all ceramic crowns,

- repair of crown margins through a minimal invasive approach,

- tunnel preparations,

- Elimination of extrinsic stains,

- cleaning of tooth surfaces before adhesive bonding, and

- Abrasion of internal surfaces of indirect tooth coloured restorations before adhesive bonding.

Recently electro medical systems (EMS) have launched AIR flow HANDY systems which use sodium bicarbonate $\left(\mathrm{NaHCO}_{3}\right)$ as the abrasive. The powder travels through the narrow nozzle of a specially delineated hand piece with the aid of compressed air. This system is indicated to eliminate biofilm, superficial stains, and young calculus on natural tooth surface, implants and restorations. It can overcome the limitations seen with rubber cups and polishing paste such as inaccessibility of areas like-

- interdental spaces of malaligned teeth

- $\quad$ Exposed tooth necks

- Pits and fissures

- Orthodontics brackets

Other advantages are that it cleans and polishes in a single sitting. When seen by its clinical relevance with regards to restorative dentistry, low abrasive powder air polishing technique is more useful for frequent cleaning of discoloured restorations. ${ }^{23}$ 

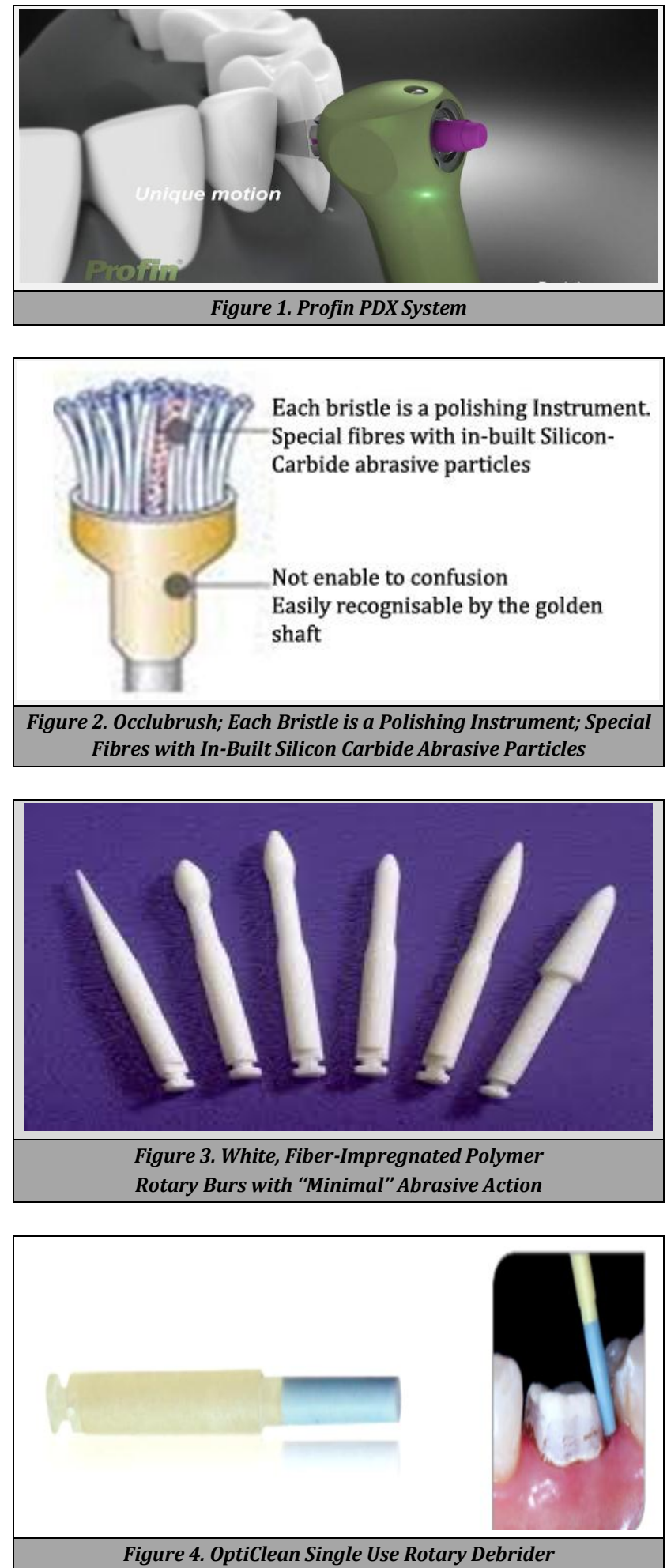

\section{Profin PDX System}

Profin PDX system (Fig 1) comprises of a contra-angle handpiece using reciprocal motion with special finishing and polishing tips - Lamineers LTA (Dentatus AB, Sweden). ${ }^{24}$ This system is mainly indicated for elimination of overhangs, contouring, grinding, and smoothening of restorations particularly in the sub-gingival and interproximal regions. The handpiece and wide assorted range of colour coded abrasive tips render extensive access to inaccessible or distant intraoral regions, permitting reshaping of enamel and other materials such as porcelain, metal fillings, resin composite and silver amalgam.

The wide range of tips and their uses are described as following -

1. Standard Spatula \#LTA - (x): For gross reduction, reshaping or finishing of bulky restorations and enamel. Colour-coded grit (x) range: 150 to 15 microns.

2. Wolfram Tungsten Spatula \#LTA - $(36,38$ or 39): Superfine abrasive for finishing and polishing of all restorations and enamel. Scuffing of healthy enamel is prevented.

3. Short Spatula \#LTA - S(x): Similar to a spatula yet shorter, narrower and knife edge shaped for better access to inter - proximal regions. Colour-coded grit (x) range: 150 to 15 microns.

4. Flexible Spatula \#LTA - F(x): Flexibility allows the tip to conform to the restoration surface. Colour-coded grit $(\mathrm{x})$ range: 100 to 50 microns.

5. Paddle \#LTA - D(x): For finishing purposes, eliminates grooves and undercuts. Colour-coded grit $(\mathrm{x})$ range: 50 to 30 microns.

6. Saw Blade \#LTA - T(x): For opening incisal and gingival embrasures. Colour-coded grit (x) range: 50 and 30 microns.

7. Shoulder / Bevel Tip \#LTA - C(x): For shaping finish lines and bevels to precision. Colour-coded grit $(\mathrm{x})$ range: 50 microns.

8. Concave Spatula \#LTA - E(x): For extra fine protrusive and lateral adjustments on concave surfaces. Colourcoded grit $(\mathrm{x})$ range: 50 microns. ${ }^{25}$

\section{Abrasive-Impregnated Brushes}

These polishing brushes are available in few shapes (pointed, cup shaped), with an assortment of polymer "bristles" impregnated with different types of abrasive particles. These flexible brushes aim for reaching into the detail grooves, protrusions, patterns, and other inaccessible interproximal areas of ceramic and composite resin restorations. 3M ESPE organization holds the patent for $3 \mathrm{M}$ Sof - Lex Brush where the abrasive used in the brush is $\mathrm{Al}_{2} \mathrm{O}_{3} \cdot{ }^{12,26-29}$

The Sof-Lex finishing brush from 3M ESPE is a soft, thermoplastic polyester elastomer that is composed of aluminum oxide abrasive particles for highly effective polishing. ${ }^{30,31-33}$ The brush is detachable type from a mandrel. The Sof-Lex Finishing Brush is easy to use, one-step, autoclavable and reusable brush developed to eliminate the separate finishing and polishing procedures. The brush bristles are gentle on the gingiva and conform as they travel over for polishing the concave and convex anatomy found on posterior composite restorations resulting in a smooth polished finish. It delivers superior finish in less time and less effort making restorative procedures more efficient. ${ }^{28}$ The intro kit consists of -

- Twelve Sof - Lex Finishing Brushes

- Two Sof - Lex Finishing Brush Mandrels - Right Angle

Occlubrush (Kerr Corporation) is a combination of rigid polycarbonate fibers impregnated with silicon carbide abrasive particles. It is a one-step polishing system for all types of composite compomers, resin modified glass ionomer cements (RMGIC) and ceramic indirect restorations. The bristles' special fibre ensure it is non-destructive to tooth 
structure or to the margins of the restorations (Fig. 2) Polishing particles are already embedded in the bristles, so no paste is required.12

Research reports that the silicon carbide- impregnated bristle polishing brushes are non-destructive and maintain surface texture, smoothness, and a high gloss shine comparatively more than an extra-fine diamond coated abrasive discs (on composite resin and enamel). ${ }^{26,27,32}$ Also these could be reused with autoclaving. Polishing efficacy of these abrasive-impregnated polishing brushes is found comparable to that seen following the use of a rubber or bonded abrasive diamond polishing device. ${ }^{12}$

These polishing brushes configuration is intriguing in its methodology for giving improved "micro - access" and hence requires further investigations and clinical assessment. Currently a new abrasive known as organisilicon is under research. In comparison to $\mathrm{SiO} 2$, it is showing better wear performance of brush materials and decreased wear percentage of materials. ${ }^{34}$

\section{Rotary Polishing Tools}

Rotary tools designed on basis of a polymer or composite resin composition or matrix, with controlled grinding have recently come into limelight for their specific action on removal of surface adherent restorative materials, including composite resin and surplus cement.

Stainbuster (Danville, San Ramon, California; and Carbotech, Ganges, France) is a slow speed, rotary zircon - rich fiber - glass bur of $14 \mu \mathrm{m}$ diameter. It is designed to remove residual composite resin, coloured coatings, provisional cement without abrading or any harm to either enamel or porcelain (Fig 3).12 Other indications include:

- removal of remnants of adhesive after bracket debonding in orthodontics,

- Smoothening and softening of root surface post root planing for encouraging healing and flap re-attachment.

- Stain removal in areas of inaccessible tooth structure.

- For maintenance of implant surfaces by gentle removal of low level calculus.

The rod-shaped bur is made up of fibres of special characteristics - they are self-sharpening and maintain abrasive power i.e. every time the resin matrix gets used up, new fibres get exposed. ${ }^{31}$ OptiClean (Kerr Dental, a division of Danaher Corporation, Orange, California) (Fig 4), a latch type rotary bur of $1.65 \mathrm{~mm}$ tip diameter is composed of aromatic polyamide containing $40 \mu \mathrm{m} \mathrm{Al}_{2} \mathrm{O}_{3} .{ }^{12}$

It is the first and the smallest tool available for faster, easier, and complete removal of any residual provisional cement and chaff on dentine / tooth preparations before final cementation and making the procedure simpler by eliminating the multiple steps of surface preparation, such as a rubber cup and pumice, or use of hand instruments. ${ }^{35,36}$ It is a single use tool and hence is considered highly hygienic.

\section{Nanotechnology Liquid Polish}

This new paint - on - polish adds a smooth, dazzling finish to any composite or temporary restoration. Nano - Beautiful or Lasting Touch (Dentsply caulk) is a recent paint on polish technique that provides smoother surfaces when used after finishing and polishing. A study conducted by D Atabek et al. concludes to have better results and positive effects on surface topographic properties with the application of nanotechnology liquid polish along with other finishing and polishing procedures. ${ }^{37}$ Although very little literature exists to support as evidence and hence further evaluation is required.

\section{CONCLUSIONS}

Finishing and polishing procedure is a significant step to achieve successful restorations clinically. The type of abrasive being used, its properties, and the material being abraded are some significant properties affecting the process of abrasion. Finishing and polishing is a guided procedure which follows a sequence descending from coarse abrasives to fine abrasives to achieve mirror like polish. Clinically the rate of abrasion is easier to manage in terms of speed in comparison to the pressure. One must keep in mind to avoid over finishing margins and contours of restorations and to abstain from overheating. Adoption of a definite sequence is the key factor in finishing and polishing of each restoration. The objective of any new office technology or innovation is to accelerate the workflow process, giving clinicians a definite asset - additional time - to concentrate on and deliver the precision. The knowledge of these new emerging concepts in restorative dentistry helps clinicians take advantage of the latest technological trends to ensure growth and stay competitive.

Financial or other competing interests: None.

Disclosure forms provided by the authors are available with the full text of this article at jemds.com.

\section{REFERENCES}

[1] Anusavice KJ. Phillips science of dental materials. $12^{\text {th }}$ edn. Philadelphia: W.B. Saunders 2012.

[2] Dutra DAM, Pereira GKR, Kantorski KZ, et al. Does finishing and polishing of restorative materials affect bacterial adhesion and biofilm formation? A systematic review. Oper Dent 2018;43(1):E37-52.

[3] Nasoohi N, Hoorizad M, Tabatabaei SF. Effects of wet and dry finishing and polishing on surface roughness and microhardness of composite resins. J Dent (Tehran) 2017;14(2):69-75.

[4] Remond G, Nockolds C, Philips M, et al. Implications of polishing techniques in quantitative $\mathrm{x}$-ray microanalysis. J Res Natl Inst Stand Technol 2002;107(6):639-62.

[5] Bashetty K, Joshi S. The effect of one-step and multi-step polishing systems on surface texture of two different resin composites. J Conserv Dent 2010;13(1):34-8.

[6] Senawongse P, Pongprueksa P. Surface roughness of nanofill and nanohybrid resin composites after polishing and brushing. J Esthet Restor Dent 2007;19(5):265-75.

[7] https://leenadentalcare.wordpress.com/2011/06/05/ finishing-and-polishing/.

[8] Filho HN, D'Azevedo MTFS, Nagem HD, et al. Surface roughness of composite resins after finishing and polishing. Braz Dent J 2003;14(1):37-41.

[9] Bollen CM, Lambrechts P, Quirynen M. Comparison of surface roughness of oral hard materials to the threshold 
surface roughness for bacterial plaque retention: a review of the literature. Dent Mater 1997;13(4):258-69.

[10] Reis AF, Giannini M, Lovadino JR, et al. Effects of various finishing systems on the surface roughness and staining susceptibility of packable composite resins. Dent Mater 2003;19(1):12-8.

[11] Morgan M. Finishing and polishing of direct posterior resin restorations. Pract Proceed Aesthet Dent 2004;16(3):211-7.

[12] Jeffries SR. Abrasive finishing and polishing in restorative dentistry: a-state-of-the-art review. Dent Clin North Am 2007;51(2):379-97.

[13] Gulati GS, Gulati NK. The effect of different polishing system on the surface roughness of a composite materials. Journal of Head \& Neck Physicians and Surgeons 2014;2(2):54-64.

[14] Rémond G, Nockolds C, Phillips M, et al. Implications of polishing techniques in quantitative $\mathrm{x}$-ray microanalysis. J Res Natl Inst Stand Technol 2002;107(6):639-62.

[15] Jeffries SR. The art and science of abrasive and finishing and polishing in restorative dentistry. Dent Clin North Am 1998;42(4):613-28.

[16] O'Brien WJ. Dental materials and their selection. In: O'Brien WJ, ed. Chap. 10. Abrasion, polishing and bleaching. Chicago: Quintessence Books 2002:156-64.

[17] Rashid H. Evaluation of the surface roughness of a standard abraded dental porcelain following different polishing techniques. Journal of Dental Sciences 2012;7(2):184-9.

[18] Ikeda M, Martin K, Nikaido T, et al. Effect of surface characteristics on adherence of $\mathrm{S}$. mutans biofilms to indirect resin composites. Dent Mater J 2007;26(6):91523.

[19] Jack L. Ferracane, materials in dentistry: principles and applications. $2^{\text {nd }}$ edn. Williams and Wilkins 2001.

[20] Craig RG. Dental materials: properties and manipulation. $8^{\text {th }}$ edn. St. Louis, Mo.: Mosby 2004

[21] Mahalaxmi S. Materials used in dentistry. Chap. 10. $1^{\text {st }}$ edn. Finishing and Polishing. India: Wolters Kluwer India Pvt Ltd 2013:539-60.

[22] Hegde VS, Khatavkar RA. A new dimension to conservative dentistry: air abrasion. J Conserv Dent 2010;13(1):4-8.
[23] Rainey JT, et al. Air abrasion: an emerging standard of care in conservative operative dentistry. Dent Clin North Am 2002;46(2):185-209.

[24] Iovan G, Stoleriu S, Nica I, et al. Surface characteristics of restorative composite resins after polishing with profine lamineer tips. Materiale Plastice 2016;53(4):755-8.

[25] https://dentatus.com/products/finishingpolishing/lamineer-tips

[26] Aschmann F, Von Weissenfluh BA. Brush for use in restorative dentistry. US Patent 6,312,257. November 6, 2001.

[27] Dubbe JW, Lund YI. Dental handpiece brush and method of using the same. US Patent 6,554,614. April 29, 2003.

[28] 3M ESPE. 3M Worldwide. http:/www.3m.com/espe. Accessed December 16, 2006.

[29] Watanabe T, Miyazaki M, Takamizawa T, et al. Influence of polishing duration on surface roughness of resin composites. J Oral Sci 2005;47(1):21-5.

[30] Yap AU, Lye KW, Sau CW. Surface characteristics of toothcolored restoratives polished utilizing different polishing systems. Oper Dent 1997;22(3):260-5.

[31] Babina K, Polyakova M, Sokhova I, et al. The effect of finishing and polishing sequences on the surface roughness of three different nanocomposites and composite/enamel and composite/cementum interfaces. Nanomaterials (Basel) 2020;10(7):1339.

[32] Nithya K, Sridevi K, Keerthi V, et al. Evaluation of surface roughness, hardness and gloss of composites after three different finishing and polishing techniques: an in vitro study. Cureus 2020;12(2):e7037.

[33] Krejci I, Lutz F, Boretti R. Resin composite polishing-filling the gaps. Quintessence Int 1999;30(7):490-5.

[34] Lin X, Liu R, Chen J, et al. Study on current-carrying friction and wear properties of copper-graphite brush material reinforced by organosilicon. Journal of Materials Research and Technology 2021;12:365-75.

[35] Bachmann MW, Bachmann S, Bachmann N. Hygiene instrument for cleaning and polishing the surface of the teeth and the composite materials of dental filings, in the shape of a rod. US Patent 6,386,874. May 14, 2002.

[36] Salem G. Dental debridement method and tool therefore. US Patent 5,882,201. March 16, 1999.

[37] Atabek D, Sillelioglu H, Olmez A. The efficiency of a new polishing material: nanotechnology liquid polish. Oper Dent 2010;35(3):362-9. 